David Isaí Lara Prieto 


\section{El problema del radicalismo social}

\section{Introducción}

En nuestra cultura actual, se promete igualdad en un sistema donde nadie es rechazado, ya que se supondría que todos tienen las mismas capacidades. El problema surge cuando, dejando de lado estas posturas superficiales enfocadas a tareas sencillas y básicas del intelecto, se toma consciencia de las diferencias que existen entre nosotros y se empieza a cuestionar esta idea tergiversada de una igualdad entre los ciudadanos.

En este texto se tratará de delimitar las diferencias entre una igualdad que proporciona el Estado a través de los derechos como parte de éste y un individualismo que marca a cada persona como ser autónomo dentro de la sociedad. Se propondrá también una definición más apropiada para la igualdad que se busca entre los habitantes de una misma comunidad y se tratarán los problemas que se presentan al darle una definición equivocada a estos términos.

También se abordarán los problemas surgidos por la igualdad cuando es confundida con la homogeneidad, al sugerir y pretender que no existen diferencias entre los individuos de una comunidad, señalándolos como iguales en todas sus características. Se analizará la problemática con los estereotipos cuando son malentendidos, al momento de catalogar individuos de distintos grupos, marcándolos por presuposiciones que no están fundamentadas.

\section{Estratificación social: el individuo en una supuesta sociedad homogénea}

En la sociedad actual existe una propuesta que es entendida por la mayoría de las personas como la igualdad de todos los individuos que la componen; pero si por igualdad se entiende la completa desaparición de las diferencias entre las personas, se identificará casi de 
manera inmediata que no puede existir una sociedad en la que todos los individuos tengan las mismas características, sencillamente porque no existen dos personas con esta cualidad común.

El conocer las diferencias entre lo que se puede y no se puede aceptar como igual dentro de la sociedad será fundamental para definir los roles y las virtudes aceptadas por la comunidad y, por contraparte, si en ésta es posible poner en práctica un individualismo radical.

Ahora bien, por postura individualista radical entenderemos la adoptada por un miembro de la sociedad que suele actuar aisladamente, sin depender de ninguna norma social. El sujeto que actúa de esta forma se vería continuamente reprendido por el sistema que se encargue de regular el comportamiento de la sociedad en la que viva. Una persona con una postura individualista radical no podría desarrollarse dentro de la sociedad, ya que ésta optaría por corregirlo o aislarlo. Por lo que en algún momento tendrá que verse involucrado en alguna actividad que lo relacione con los demás.

En el escrito, no intentaremos hablar de personas meramente individualistas, ya que sencillamente no se encuentran de forma activa dentro de la sociedad; por el contrario, se intentará prestar atención a quienes su conducta les permita desarrollarse de una manera paralela a los de su mismo contexto. Lo que consideraremos en el ensayo será un individualismo reducido de su postura radical y que servirá al sujeto como una herramienta para definirse.

Por otro lado, la igualdad no estará llamada a ser confundida con la homogeneidad y cabe señalar que no existen diferencias entre los individuos que conforman la sociedad. La definición de igual nos da a entender algo que tiene la misma naturaleza, calidad o cantidad semejante, y que es de la misma clase o condición. No se debe confundir la igualdad con la homogeneidad dentro de la completa desaparición de las características individuales, sino que deberá entenderse como las semejanzas que los individuos comparten dentro de la misma comunidad.

Como señala Luis Villoro en Estado plural. Pluralidad de culturas, los rasgos que comparten los individuos de una comunidad los hacen parte de un mismo grupo que los identifica bajo el mismo contexto histórico y político.

En efecto, una manera general de reconocer un objeto es encontrar en él ciertos signos distintivos. [...] podríamos identificar 
la pertenencia de un pueblo por ciertos signos distintivos de su cultura.

[... Trata de encontrar en las expresiones culturales, las notas que expresen una peculiaridad nacional. Trata de encontrar en las expresiones culturales ciertos rasgos que puedan verse como característicos de esa cultura, que se prolonga desde un pasado histórico ${ }^{1}$.

Pero habrá que aclarar que el que compartan estas peculiaridades no los hará uniformes en cuanto a sus características personales; sino que solamente los pondrá dentro de un mismo contexto cultural. Dentro de esta comunidad seguirá existiendo una diferenciación entre los individuos que la componen.

El desarrollar una igualdad en nuestra sociedad no será tarea sencilla si se conoce la dificultad que representa el individualismo. Una persona no puede ser igual a otra por el mismo hecho de que no pueden compartir las mismas características. Dentro del estado, no puede haber igualdad si por ésta se entiende que no existirán diferencias.

\section{El individuo en una sociedad estereotipada}

Aquí es donde el sistema político y la estructura social deberán actuar para garantizar un trato justo para todos los que se dirigen por ellos. El modo en el que estos derechos sean concedidos de manera equitativa dependerá de la calidad de la persona que los necesite. Porque, por ejemplo, no se pueden garantizar en su totalidad los mismos derechos para hombres y mujeres, ya que el hecho de tener un organismo diferente nos crea diferentes necesidades. Es aquí donde las leyes, con el objetivo de asegurar el trato justo, se presentan como insuficientes si trataran de identificar y conocer cada caso en forma individual. El trabajo de este sistema es garantizar las necesidades básicas que tiene la comunidad y de ahí dividirla en grupos que compartan necesidades para procurarlos de una manera más eficiente. Dividiendo a la población por sus necesidades comunes, se consigue una mejora al momento de tratar grupos en lugar de individuos.

1 Villoro, Luis, Estado plural. Pluralidad de culturas, Paidos, México, 1999, p. 72. 
Afirmar que no existen diferencias entre quienes componen una sociedad es sostener que no hay características propias del individuo y que, en consecuencia, no existe una identidad propia del sujeto.

En resumen, decir que entre los miembros de una comunidad no debe haber diferencias podría dar paso a limitar el desarrollo individual de este conjunto de personas y a complicar las atenciones que el Estado les tiene que procurar. Afirmar una nula diferenciación sólo podría complicar las cosas en una sociedad donde el individuo juega un papel importante. Sería mejor aseverar que, como miembros activos de una comunidad, estamos protegidos por un sistema que procura garantizar para todos sus integrantes el trato más justo, que depende de las necesidades que éstos tengan.

\section{Estereotipos laborales}

Dejando en claro el modo erróneo en el que se puede interpretar la igualdad entre los integrantes de la comunidad, se debe impulsar el uso adecuado de ésta. Pero aquí es donde comienzan las verdaderas dificultades, porque se tendrá que empezar a generalizar sobre grupos específicos, ¿es verdaderamente esto algo malo? Si lo dejamos sin aclarar, probablemente será tomado de una mala manera y tachado como un argumento que defiende la discriminación injustificada, dado el contexto en el que habitualmente se nos presenta; pero si se aclara este punto, se entenderá por qué ciertas personas están capacitadas para trabajos específicos y otras no.

Como lo explica Homi K. Bhabha al hablar del colonialismo, los estereotipos surgen al momento de hacer las divisiones necesarias entre los miembros de la comunidad y al llevar estas etiquetas a un extremo que se encarga de fomentar la discriminación y segregación de ciertos grupos. Con éstos se propone una excusa perfecta para la discriminación si nunca son cuestionados. En este caso de radicalismo no se necesita ninguna prueba para demostrar que lo que dice el estereotipo dentro de la tradición está bien.

De igual manera, el estereotipo, que es la mayor estrategia discursiva del colonialismo es una forma de conocimiento e identificación que habla de algo que está siempre en "su lugar", que está ya bien conocido, y sobre algo que se debe repetir con ansiedad... 
así como la duplicidad esencial del asiático o el bestial permiso sexual del africano que no necesitan prueba, que en realidad nunca, durante un discurso, son probados ${ }^{2}$.

Pongamos un ejemplo sencillo: se tiene a dos sujetos para hacer dos trabajos, de modo que cada uno tendrá que realizar uno de ellos. El primer sujeto es robusto y capaz de levantar objetos pesados sin mayor dificultad; pero es torpe en sus movimientos y se le dificulta el trabajo manual debido a la constitución de su cuerpo. El segundo sujeto es delgado, ágil y flexible; aunque no es capaz de levantar objetos muy pesados, ya que la constitución de su cuerpo no se lo permite. Una tarea consiste en levantar grandes pesos y la otra en realizar una actividad manual y delicada. Será obvio a primera vista quién es el indicado para cada uno de los trabajos, porque las capacidades de uno lo hacen más apto para determinadas labores y las del otro lo califican mejor para cumplir con facilidad la otra tarea.

En este ejemplo, no habría razón para pensar que el designar tareas específicas debido a la condición y capacidades de las personas es un acto discriminatorio, pues depende de éstas el ejecutar un trabajo acorde a lo que nos sea más fácil. En razón de esto, el responsable de organizar y repartir las labores tendrá que buscar lo más adecuado para cada sujeto, partiendo de que quien sea el más apto para la labor sea el que la realice.

$\mathrm{Si}$, por el contrario, seguimos un modelo donde las tareas se reparten de manera imparcial, podría darse que nuestros sujetos tengan que realizar una tarea que, en lugar de buscar para ellos lo que sería más justo y sencillo dadas sus capacidades, proponga el encargarles algo en lo que tendrán más dificultades. Teniendo en cuenta las capacidades de cada individuo, veremos que existen ciertas personas que están mejor capacitadas para realizar ciertas actividades, y eso pasa, de igual manera, con los grupos de personas. Dado que si los individuos comparten características que los capacitan para realizar de manera adecuada una tarea, lo ideal será que se les procure la labor acorde a sus capacidades. Lo mismo pasará con otro grupo que carezca de las capacidades para realizar cierta tarea, ya que se deberían ubicar en labores acordes a sus capacidades.

¿Qué se debe buscar al desempeñarse como profesional?

2 Cfr. Homi K. Bhabha, The location of culture, Routledge, Great Britain, 1994, p. 66 . 
Parecerá obvio que se tiene que adecuar el individuo a su trabajo, partiendo de la vocación y las capacidades de éste. Por ello, hacer distinciones entre grupos debido a las cualidades compartidas por sus integrantes no tendrá que verse de manera negativa, ya que esto procura un mejor trato, consecuente de una mejora en la organización. No parece correcto sugerir que todos pueden hacer las mismas labores, siendo que éstas dependen de capacidades sobre las que no tenemos un control total.

\section{Radicalismo social: sociedad uniforme y discriminación}

Aparte de la mala interpretación que se puede dar al sentido de ser igual a otro individuo y al derecho de ser tratados con justicia, se presentan otros problemas que se desglosan de estos anteriores, llevando los estereotipos y la homogeneidad a sus extremos. Cabe aclarar que éstos no son los únicos problemas presentes en la comunidad, pero son de especial importancia al momento de designar el espacio que le corresponde a cada uno de los individuos.

El primer problema por abordar se plantea como la erradicación de las diferencias de los integrantes de la comunidad. El actual movimiento inclusivo pretende que todos los seres humanos sean juzgados como iguales, independientemente de su orientación sexual, su identidad más allá de la condición en la que nacieron, su color de piel y su religión, entre otros. El noble ideal propone que no habría razones para prejuzgar a nadie; pero como parece pasar con la mayoría de las propuestas adoptadas por un pueblo poco crítico, se termina por tergiversar la propuesta inicial al punto de olvidar los principios de respeto que están defendiendo, adoptando una postura radical.

La propuesta errónea en un movimiento como éste nace a partir de la ignorancia de individuos que, al portar la bandera del grupo al que pretenden representar, terminan por desprestigiar los valores que se pretendían defender en un principio. El problema no es el movimiento ni lo que propone, el problema es la gente que no está capacitada para argumentar en favor de su verdadero motivo, que termina por malinterpretar el mensaje y desinformar a quienes deciden pertenecer a un movimiento ya degradado en sus valores.

El segundo problema, presente en los estereotipos, se basa en los mismos principios, pero está enfocado a una postura dife- 
rente, puesto que, efectivamente, un estereotipo es una especie de conocimiento que se transmite de generación en generación dependiendo del contexto de la sociedad en la que se dé. Lo que orilla a alguien a usarlos de un modo inapropiado, por no decir racista, será el sentido y el uso que les dé. Esto pasa con todo el conocimiento, pues éste no es malo, sino que lo juzgamos como malo en la medida del uso que se le dé.

Lo que provoca que se desarrollen estas conductas intolerantes e injustificadas es la inflexibilidad que se tiene al momento de cuestionar las posturas que están presentes en las tradiciones. En esencia, un estereotipo no será malo si va de acuerdo con la verdad y los hechos, pues es comprensible que se tenga un acuerdo colectivo en ciertas cosas si éstas actúan de una manera determinada y predecible. Por ejemplo, lo sería el hecho de que, ya que en los hombres la densidad ósea y la masa muscular es mayor, se tenga una especie de conocimiento en forma de estereotipo que nos diga que la generalidad de los hombres es más fuerte físicamente que la que constituye la generalidad de las mujeres. Un estereotipo habla en la mayoría de los casos de partes que están dadas ya en la naturaleza y que constituyen nuestro ser más básico.

Ahora bien, lo que termina por demarcar a los estereotipos dentro de una postura "mala" es atribuirles características falsas a grupos en concreto y aceptarlas sin hacer cuestionamientos sobre éstas. El mismo principio de ignorancia presente en el problema de la homogeneidad se encuentra aquí al no tratar de una manera adecuada el principio por el cual se fundó esta idea, desvirtuándola en beneficio de otros.

\section{Conclusiones}

A modo de conclusión podemos ver que a este problema se le puede dar una solución sencilla, y es el no permitir que se considere como aptos para tratar los temas a quienes no están capacitados ni en disposición para argumentar en favor de obtener la verdad, y que en cambio buscan un beneficio único para un grupo de individuos. Abordar temas sobre los que no estamos informados y tratarlos desde una postura poco crítica fomenta el aceptar premisas equivocadas, lo que da lugar a conclusiones erróneas. Los temas que tratan sobre el bien común deben ser desarrollados por gente 
preparada en él, pues está de más decir que, si se desconoce del tema, la opinión tiende a ser errada.

El fomento de las virtudes, desde una edad temprana, será de gran ayuda para erradicar el problema que la intolerancia y la ignorancia terminan por agraviar; aunque, debido a las distintas capacidades que hay entre los individuos, siempre habrá sujetos a los que se les facilite más esta labor. Por lo que no es lo ideal el dejar que alguien que desconoce del tema tome decisiones en cuanto al funcionamiento y el camino a tomar del tema en cuestión.

Y entonces, ¿qué clase de individualismo se presentará como más adecuado para la cultura en la que nos encontramos? Será donde el individuo reconozca que entre él y los demás existen diferencias que lo marcarán como único en cuanto está solo dentro del paréntesis que es cumplir con todas sus características. Reconocerse como individuo no deberá ser factor para que se produzca un alejamiento de la sociedad, sino todo lo contrario, esta diferencia ayudará a la construcción de una sociedad mejor, siempre y cuando se adopten las características individuales.

Así, dentro de un sistema que procure lo mejor para sus integrantes y que inculque en ellos el respeto hacia las virtudes humanas, se podría llegar a desarrollar una cultura inclusiva. 
